RESEARCH PAPER RP1159

Part of Journal of Research of the National Bureau of Standards, Volume 21, December 1938

\title{
TRENDS OF CHARACTERISTICS OF THE IONOSPHERE FOR HALF A SUNSPOT CYCLE
}

\author{
By Newbern Smith, Theodore R. Gilliland, and Samuel S. Kirby
}

\section{ABSTRACT}

The increase of solar activity from the sunspot minimum of 1933 to 1938 resulted in large increases in the ionization of the upper atmosphere. During this time the ionization densities of the $E$ layer increased by a ratio of approximately 1.55 to 1 and those of the $F_{2}$ layer by about 4 to 1 . Superposed on this longperiod increase were the more or less regular diurnal and seasonal variations. Graphs indicating the long-time variations for the different layers at several different times of day are shown.

\section{CONTENTS}

Page

I. Introduction

II. Diurnal and seasonal variations _.

III. Long-period variations

IV. Conclusion

\section{INTRODUCTION}

A program of observations extending over the past 5 years has yielded information on the characteristic regular variations of the critical frequencies and virtual heights of the various layers of the ionosphere. These regular variations are diurnal, seasonal, and from year to year. The present paper summarizes this information and indicates the trend and probable cause of the year-to-year variation.

Since the ionization of the earth's upper atmosphere is due, wholly or principally, to energy from the sun, changes in solar activity might be expected to produce corresponding changes in the ionization of the ionosphere. Sunspot numbers are a rough indication of average solar activity. The critical frequencies and virtual heights, which are directly related to the ionization of the ionosphere, might therefore be expected to vary with the sunspot numbers.

Sunspots are but one manifestation of the solar activity influencing the sun's ultraviolet radiation, which is in turn responsible for the ionization of the ionosphere. There is, therefore, no reason to expect detailed correlation between critical frequencies and sunspot numbers. In the absence of a better criterion of solar activity, however, it may be assumed that sunspot numbers give, for long-time averages but not in detail, an index of the intensity of solar radiation. It might, therefore, be expected that the ionosphere critical frequencies 
and the sunspot numbers would follow the same trend, but not necessarily show complete correlation.

Regular ionosphere observations of the National Bureau of Standards have covered a period, from 1933 to date (1938), of about half a sunspot cycle. ${ }^{1}$ Less regular observations were made from 1930 to 1933. During the half-cycle from 1933 to 1938 the general solar activity, as measured by the sunspot numbers, increased from a minimum in the fall of 1933 to what appears to have been a maximum in 1937 , and the ionization of the various layers increased accordingly.

\section{DIURNAL AND SEASONAL VARIATIONS}

The ionosphere observations of the National Bureau of Standards have indicated the regular diurnal and seasonal characteristics of the critical frequencies and virtual heights of various layers.

Figure 1 shows typical average diurnal curves of critical frequencies and virtual heights for winter and summer conditions, represented by the months of December 1936 and June 1937, respectively. The vertical dashed lines are for the times of sunrise and sunset at the ground (not in the ionosphere). The general diurnal and seasonal variations shown in figure 1 have repeated themselves so consistently as to leave little doubt that they are characteristic of the ionosphere at the latitude of Washington.

No regular diurnal or seasonal variation has been observed in the minimum virtual height of the $E$ region, which appears to be always between 110 and $120 \mathrm{~km}$. The critical frequency, $f_{z},{ }^{2}$ for the ordinary ray reflected from the $E$ layer, has, however, a regular diurnal and seasonal variation. As may be seen from figure $1, f_{s}$ varies synchronously with the altitude of the sun, reaching the diurnal maximum at noon, and having a higher diurnal maximum in the summer than in the winter. This behavior accords with the simple theory of ionization by ultraviolet light from the sun, according to which, ${ }^{3}$ assuming recombination to be sufficiently rapid,

$$
f_{B}=K \cos ^{1 / 4} \psi
$$

where $\psi$ is the zenith angle of the sun, and $K$ is a factor depending on the intensity of the solar radiation.

It has been established that, for values of $\psi$ not too close to $90^{\circ}, f_{B}$ is proportional to $\cos ^{1 / 4} \psi$, within fairly close limits, throughout the daylight hours of any one day. This would indicate (1) that the $E$ layer is ionized principally by ultraviolet light from the sun, and (2) that $K$ is very nearly a constant throughout the day, that is, the ionizing radiation from the sun does not vary much in the course of a day. It will be noted also, from figure 1 , that the $f_{z}$ begins to rise about a half hour before ground sunrise.

\footnotetext{
1 T. R. Gilliland, Multifrequency ionosphere recording and its significance, J. Research NBS 14, 283 (1935 RP769. Proc. Inst. Radio Engrs. 23, 1076 (1935).

T. R. Gilliland, S. S. Kirby, N. Smith, and S. E. Reymer, Characteristics of the ionosphere and their application to radio transmission, J. Research NBS 18, 645 (1937) RP1001; Proc. Inst. Radio Engrs. 24, 823 (1937).

T. R. Gilliland, S. S. Kirby, N. Smith, and S. E. Reymer, Characterisics of the ionosphere at Washington, $D$. C. Series of monthly reports beginning in the Sept. 1937 issue of the Proc. Inst. Radio Engrs. 8 The notation is that used in the second paper of reference 1.

P. O. Pederson, Propagation of Radio Waves. Chap. V. \& VI.

I S. S. Kirby and E. B. Judson, Recent studies of the ionosphere, J. Research NBS 14, 469 (1935) RP780; Proc. Inst. Radio Engrs. 23, 733 (1935).
} 
No such simple relation as that given above has characterized the variation of the critical frequencies of the $F, F_{1}$, and $F_{2}$ layers. As may be seen from figure 1, the daytime critical frequencies of the upper layer are much higher in the winter than in the summer, although the night critical frequencies are somewhat lower. The diurnal maximum of the $f^{x} F_{2}$ almost always occurs after noon, and is much later in the day in the summer than in the winter. The post-sunset fall in the night $f_{F}^{x}$ is much more rapid in the winter than in the summer; indeed
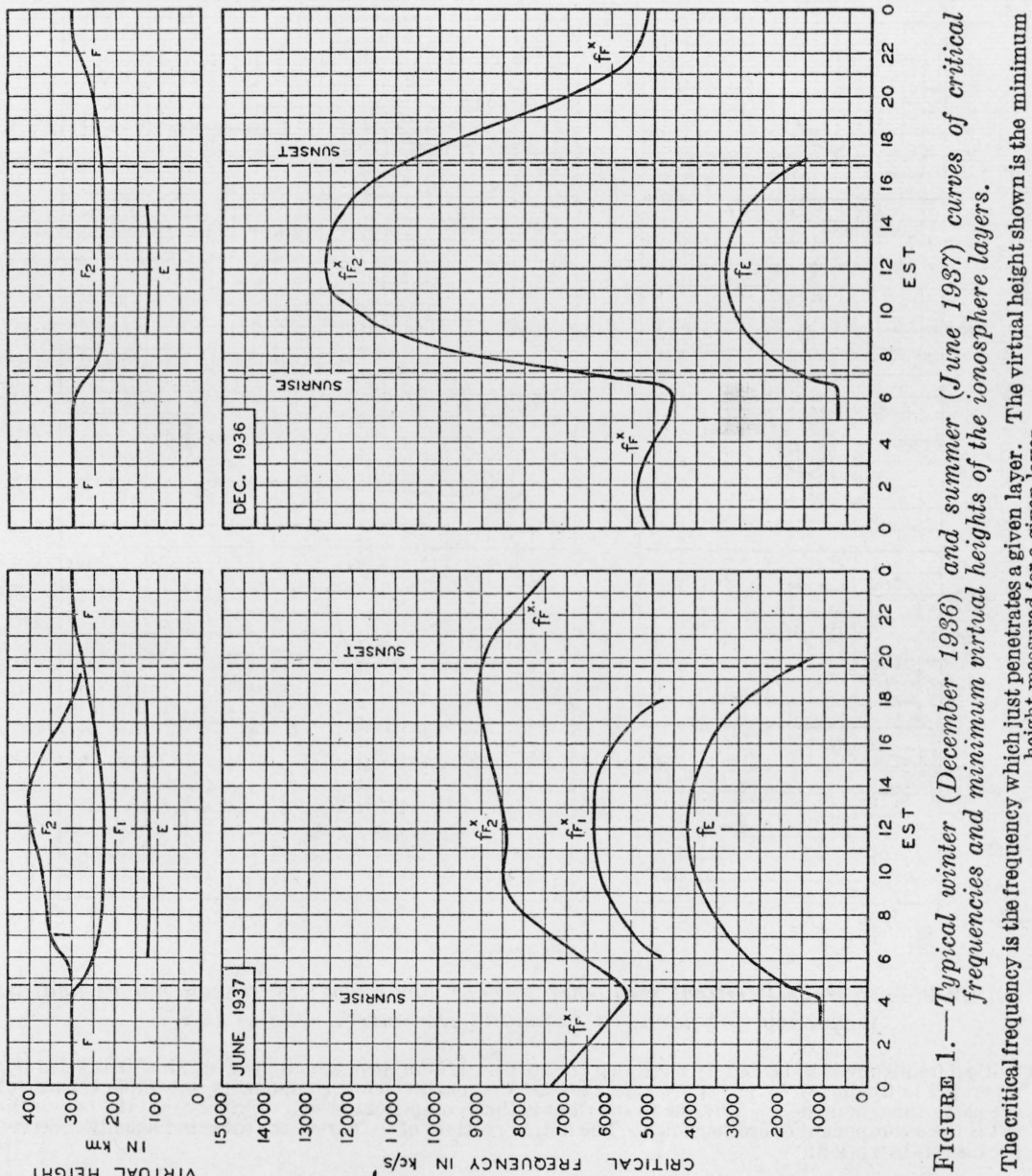

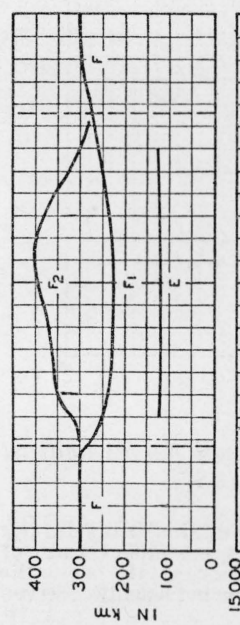

$\perp$ Н

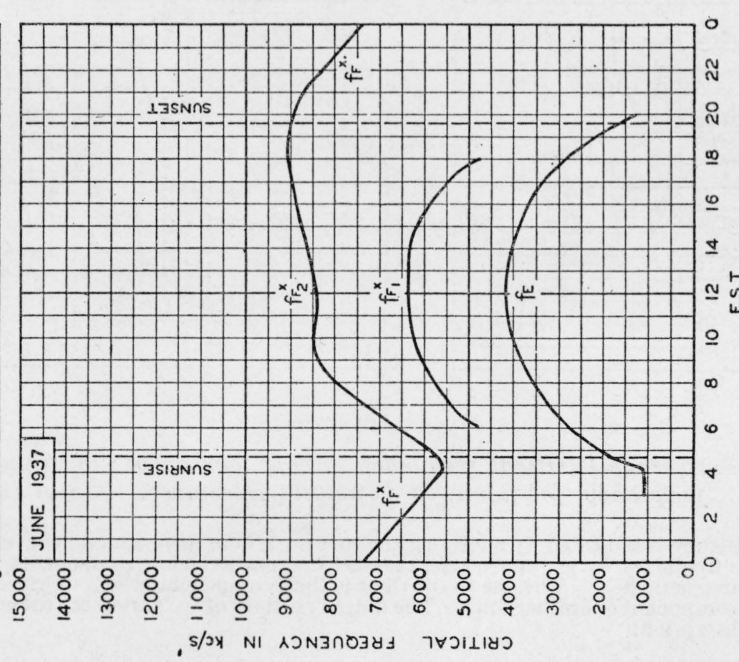

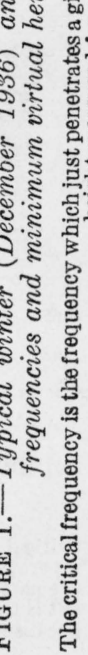

the midnight $f_{F}^{x}$ in the summer is often as high as or higher than the noon $f_{F_{2}}^{x}$. The outstanding characteristics of the $f^{x}$ and $f^{x} F_{2}$ are, then, (1) a seasonal variation inverse to that of the $f_{B}$, and (2) a diurnal lagging behind the altitude of the sun.

Like the $f_{E}$, the $f_{F_{2}}$ begins to rise from its diurnal minimum about a half hour before ground sunrise. No satisfactory quantitative theory has thus far been advanced to account for this pre-sunrise increase of ionization. It seems possible that the explanation may lie in a scattering of ultraviolet radiation from, or a refraction of this radiation in 
the upper ionosphere. Any refraction would have to be caused by some kind of anomalous dispersion, inasmuch as the frequency of the ultraviolet radiation in question lies in a region of absorption for some part of the ionosphere. The fact that both the $f_{E}$ and $f_{F_{2}}$ start to increase simultaneously would seem to be evidence in favor of ultraviolet light as the ionizing agent of the $F_{2}$ region.

The fact that $h_{F_{2}}$ is much higher during the summer day than during the winter day is evidence for the existence of a temperature effect
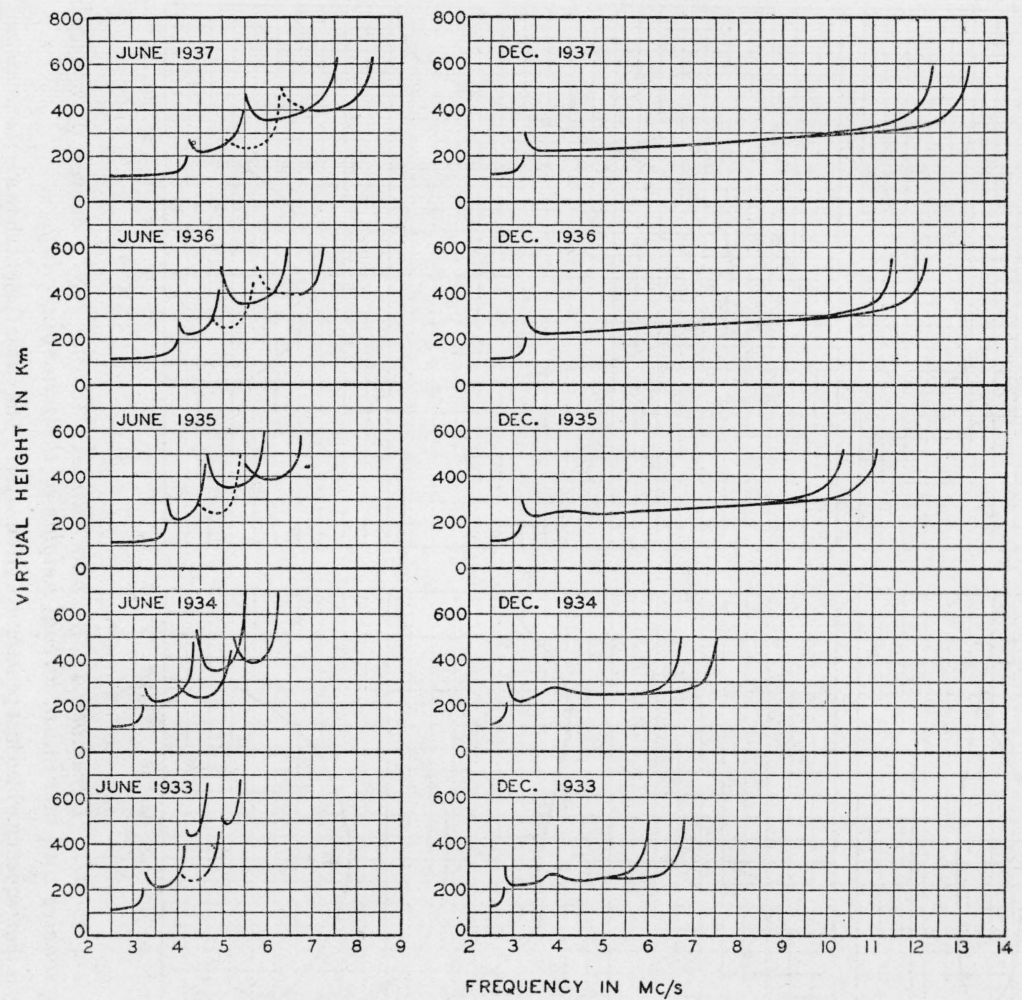

FIGURE 2.-Average "critical frequency sweeps" or curves of virtual height against frequency, for noon, in June and December, 1933 to $193 \%$.

A critical frequency is indicated by a cusp on the curve. Where there are two curves, similar in form but separated in frequency by about $800 \mathrm{kc} / \mathrm{s}$, they are the magneto-ionic components, separated because of the earth's magnetic field. The one to the right is the $x$-component or extraordinary ray; the one to the left is the o-component or ordinary ray. The dotted portions of the curves are those not usually observed because of absorption.

in the $F_{2}$ region. ${ }^{5}$ It has been pointed out ${ }^{6}$ that such a temperature effect may be in part responsible for the lower $f_{F_{2}}^{x}$ in the summer than in the winter.

The $h_{F}$ is usually about $300 \mathrm{~km}$ during the night and early morning, both in winter and in summer, and starts to fall to the $h_{p_{1}}$ level at about the time $f_{F}^{x}$ starts to increase, that is, about a half hour before

\footnotetext{
B E. O. Hulburt, Analysis of recent measurements of the ionosphere, Phys. Rev. 46, 822 (1934); Theory of the ionosphere, Terr. Mag. 40, 193 (1935).

$6 \mathrm{~L}$. V. Berkner and H. W. Wells, Non-seasonal change of $F_{s}$ region ion density, Terr. Mag. 43, 15 (1935).
} 
sunrise. This pre-sunrise fall is rapid, but the post-sunset rise in $h_{r}$ is gradual, corresponding to the relatively slow decrease of $f_{F}^{x}$ during the same period:

The stratification of the $F$ layer into $F_{1}$ and $F_{2}$ layers occurs distinctly only during the summer day, but there is reason to believe that it exists also latently during the winter day. ${ }^{7}$ No stratification of the $F$ region into $F_{1}$ and $F_{2}$ layers has been observed at night.

It should be noted that radio-transmission conditions in general follow the critical frequencies. When the critical frequencies are high the maximum usable frequencies are high and the best frequencies for transmission are also high.

\section{LONG-PERIOD VARIATIONS}

Figure 2 shows typical average curves of frequency against virtual height for June and December, representing summer and winter conditions, from 1933 to 1937, inclusive. Increase of the critical frequencies with the increase in solar activity has been relatively large for the $F_{2}$ region, and smaller but well marked for the $E$ and the $F_{1}$ regions. The virtual heights and general form of the curve of frequency against height have remained the same. The chief difference, aside from the magnitude of the critical frequencies, has been a tendency toward disappearance of the daytime $F$-layer stratification, especially during the winter. It will be noted that the minimum virtual heights have remained about the same during the period from 1933 to 1937.

In order to eliminate seasonal variations, 12-month running averages of critical frequencies were taken for comparison with sunspot numbers. A 12-month running average of sunspot numbers was also taken, in order to eliminate short-time variations and to exhibit the long-time trend in solar activity. The sunspot numbers used are the Zürich provisional numbers.

Another method of eliminating the seasonal variation can be employed in the case of the $E$ region. The factor $K=f_{E} / \cos ^{1 / 4} \psi$, is the value which $f_{E}$ would have if measured at the subsolar point. This quantity should thus increase with the intensity of the ionizing radiation from the sun, and should bear the same relation to the general solar activity as does the ionizing radiation. By comparing $f_{B} / \cos ^{1 / 4} \psi$ with the sunspot numbers, it should be possible to see how well the sunspot numbers indicate the intensity of the ionizing radiation responsible for the $E$ layer.

This has been done in figure 3 for the monthly averages of $f_{B}$ and sunspot numbers. The solid line in graph $\mathrm{b}$ is the monthly average noon $f_{E}$ plotted for each month since 1933. The dotted line shows the subsolar $f_{B}$ plotted for the same period as is the regular noon $f_{B}$. The correspondence of the subsolar $f_{B}$ with sunspot numbers is incomplete in some details but nevertheless impressive. It shows that there is in general a fairly close relationship between the cause of the $E$-layer ionizing energy and the cause or number of sunspots. Over the period covered by the observations, it may be concluded that there is an excellent general correlation, and even a fair amount of agreement in details between the monthly averages of $f_{E}$ and the sunspot numbers. The day-to-day correlation, however, is small.

\footnotetext{
7 T. R. Gilliland, S. S. Kirby, N. Smith, Characteristics of the ionosphere at Washington, D. C., March 1938, Proc. Inst. Radio Engrs. 26, 640 (1938).
} 
The contour diagram at the bottom of figure 3 represents a space model of $f_{B}$ (indicated in $\mathrm{Mc} / \mathrm{s}$ ) against the time of day and the month of the year, for critical frequencies above $2.6 \mathrm{Mc} / \mathrm{s}$. The regular seasonal variation is quite well marked, the values being higher in the summer than in the winter. It is interesting to note how the contours of equal $f_{B}$ follow the sunrise and sunset lines. The greater

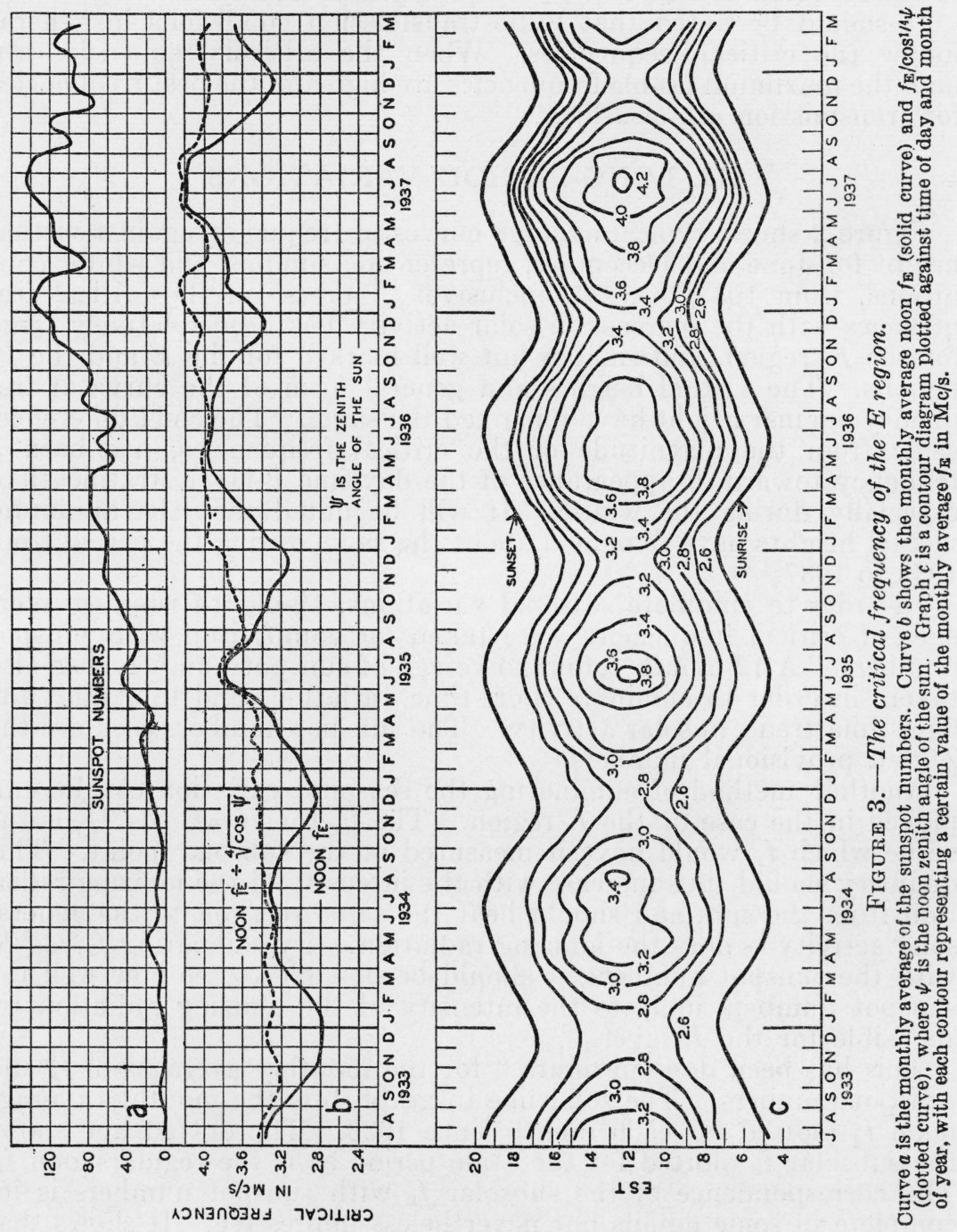

number and closer spacing of contours during the latter part of the period shows how the critical frequencies have risen with the general long-time increase in solar activity.

The $F_{2}$ layer presents a more complicated picture, since purely terrestrial influences, such as the temperature of the layer, play an important part. No such simple method therefore exists for eliminating diurnal and seasonal variations as may be done for the $E$ layer. 
Because of this difficulty in eliminating the seasonal and diurnal variations of $f_{F_{2}}$ it is hard to tell whether there is correlation in any considerable detail with sunspot numbers; such as was observed in the case of the E-region.

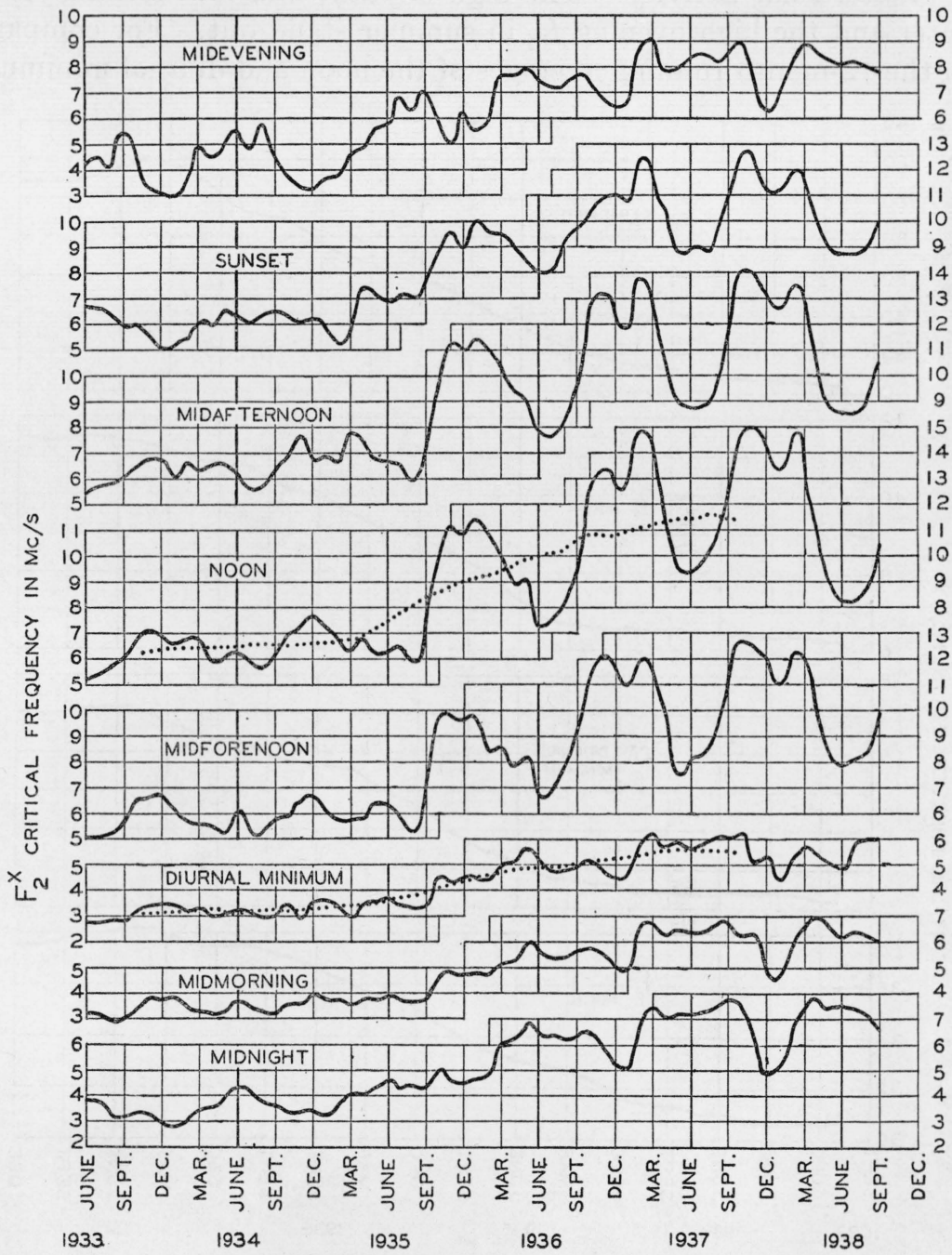

FIGURE 4.-Monthly average values of $f^{x_{F}}$ (night) and $f^{x_{F}}$ (daytime) plotted for the period 1933 to 1938.

The dotted curves are 12-month running averages for the same hours.

Figure 4 shows the variation, for each month, of the $f^{x} F_{2}$ for various parts of the day. The "midmorning" curve is for the time half-way between midnight and sunrise, and the "diurnal minimum" curve is for the time when $f_{F}^{x}$ reaches its diurnal minimum, about 30 minutes before ground sunrise. The midforenoon, midafternoon, and mid- 
evening, curves are for times half-way between sunrise and noon, noon and sunset, and sunset and midnight, respectively. All of these curves show the general rise in critical frequencies superimposed on the seasonal variation. The amplitude of the seasonal variation is greater for greater solar activity. The high daytime and low evening $f_{P_{2}}$ in winter and the high evening $f_{F_{2}}$ in summer stand out. For comparison the 12-month running averages of the noon and diurnal minimum

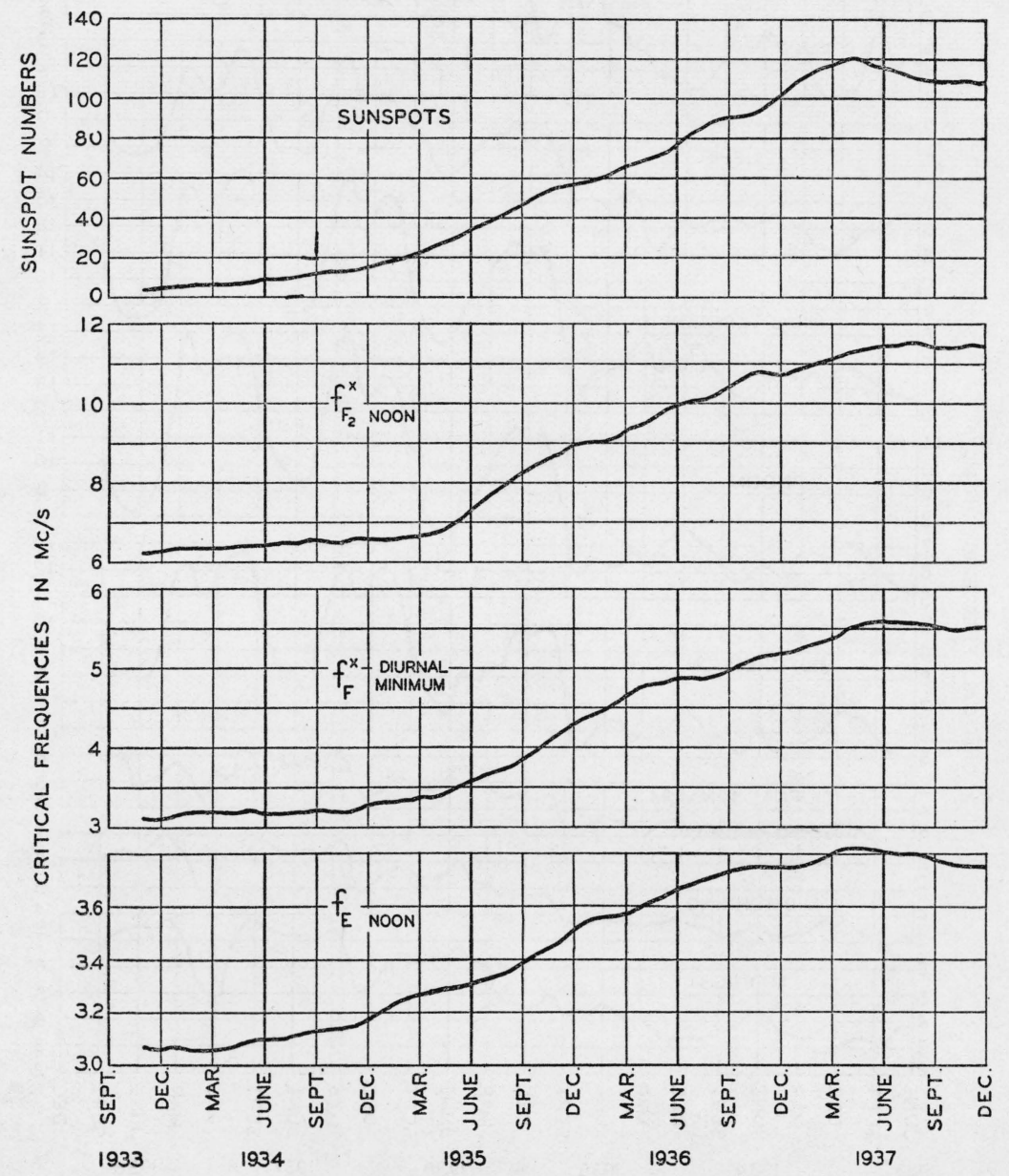

Figure 5.-Twelve-month running averages of critical frequencies and sunspot numbers.

$f_{P_{2}}^{x}$ are shown dotted to indicate the trend and the amplitude of the seasonal variation. The diurnal minimum shows the least seasonal variation; this may be more or less expected, since the direct influence of the sun would be a minimum at this time. The midwinter dip in the daytime $f_{P_{2}}$ should also be noted.

If we consider the 12-month running averages of the critical frequencies and sunspot numbers, we may see how the trend of $f_{P_{2}}$ and 
$f_{B}$ follows the trend of the sunspot numbers. Figure 5 shows these averages, for the noon $E$ layer, the noon $F_{2}$ layer, and the $F$ layer at the diurnal minimum 30 minutes before sunrise, plotted for the period from 1933 to 1937.

The diagram shows that:

1. The $f_{B}$ followed the sunspot numbers quite closely. The two quantities began to rise together, reached a maximum together, and began falling together. The rates of change also seemed to vary together.

2. The diurnal minimum $f^{x}{ }_{F}$ and the noon $f_{F_{2}}^{x}$ also followed the general trend of the sunspot numbers, but they lagged behind the sunspot numbers in starting to rise, and they kept on rising after the sunspot numbers had started to fall.

3. The sunspot-number curve showed a maximum at about April 1937. This may not indicate the major sunspot maximum of this cycle, but it is the first time since 1933 that the running average showed a decrease.

4. During the half-cycle of sunspot activity the $f_{B}$ increased by a factor of about 1.25 , and the $f^{\circ}{ }_{r}$ and $f_{P_{2}}$ by a factor of about 2.00 . The $f^{o}{ }_{F}$ and $f_{{ }_{F_{2}}}$ represent the ordinary-ray critical frequencies and are about $800 \mathrm{kc} / \mathrm{s}$ lower than the $f^{x}{ }_{r}$ and $f_{F_{2}}^{x}$, the extraordinaryray critical frequencies, which are shown in the figures. This corresponds to an increase of $E$-layer ionization by a factor of about 1.55 and an increase of $F$ - and $F_{2}$-layer ionization by a factor of about 4.00 . It is interesting that during the rise of the critical frequencies, the amplitude of the seasonal variation of $f_{B}$ increased by a factor about 1.60 and that of $f_{F_{2}}$ by a factor of about 4.00 ; this effect is shown in figures 3 and 4.

Figure 6 is an extension of a curve already published ${ }^{8}$ showing the relation between the annual averages of $f_{B}, f^{0}{ }_{F_{1}}$, and $f^{x}{ }_{F_{2}}$ and the sunspot numbers. Most of the points plotted are the same as certain points on the running average curves, except for the $f^{o}{ }_{F_{1}}$; no running average could be taken of this quantity, since it could be measured only in the summer.

The general correlation between the average of critical frequencies and sunspot numbers is impressive. It indicates that there is on the average a definite relation between sunspot numbers and the solar activity responsible for the ionization of the earth's ionosphere. This relationship seems to be much closer for $E$-region ionization. In view of the difficulty and wide variations in methods of counting sunspots, together with the lack of any distinction made between active and inactive sunspot groups, close correlation is hardly to be expected.

In order to show the correlation between sunspot numbers and critical frequencies in as complete a manner as possible, the data have been summarized and plotted in figure 7 , for which the coordinates are the 12 -month running averages of the quantities. It is seen that all the critical frequencies increased with sunspot numbers. The variations of the points from the smooth curves may well be due to an irregular relation between sunspot numbers and the solar activity responsible

${ }^{8} \mathrm{E}$. B. Judson, Comparison of data on the ionosphere, sunspots, and terrestrial magnetism, J. Research NBS 17, 323 (1936) RP913; Proc. Inst. Radio Engrs. 25, 38 (1937). 

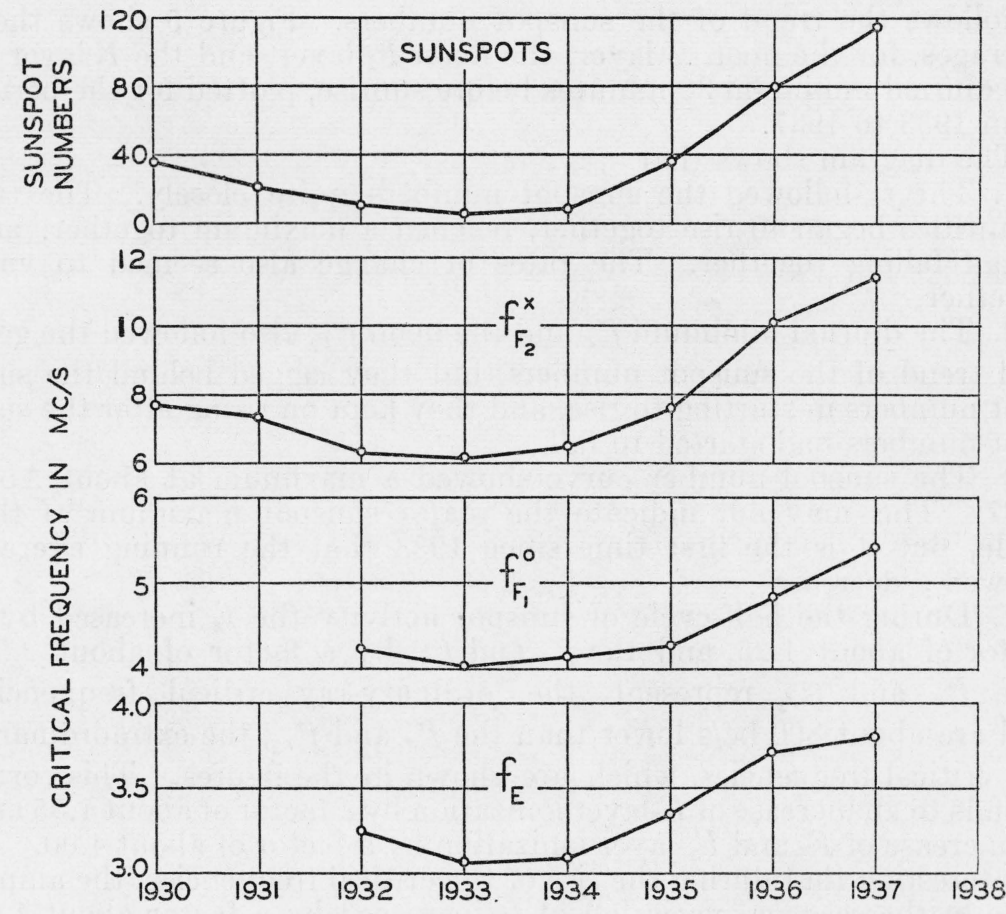

FIGURE 6.-Comparison of annual averages of sunspot numbers and noon critical frequencies of ionosphere layers.

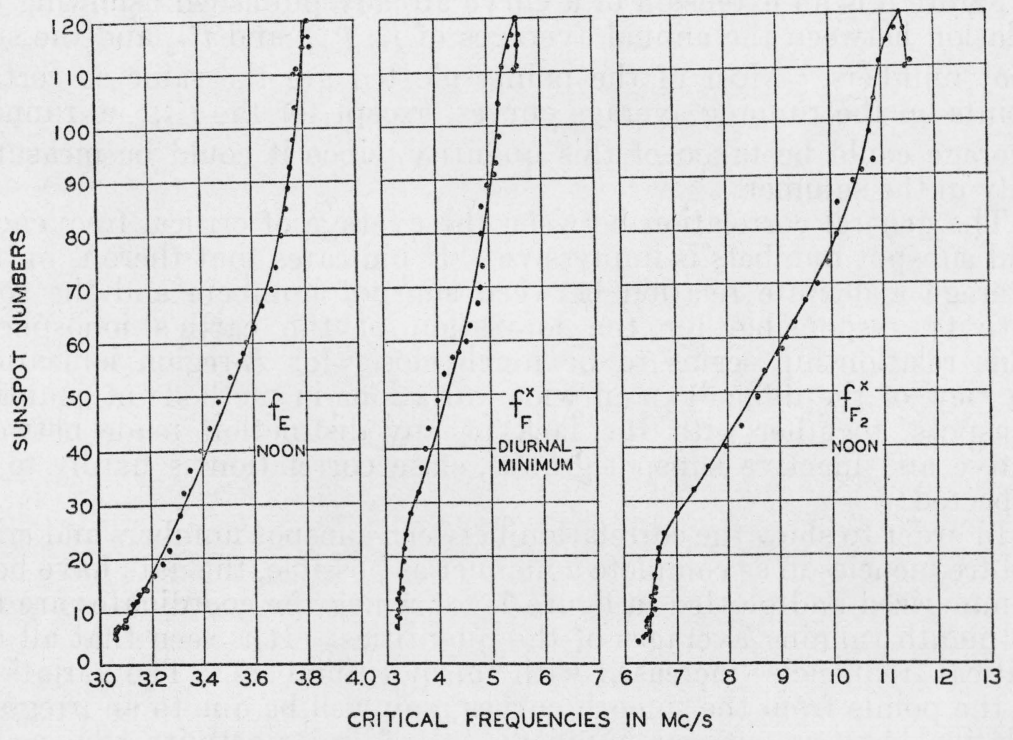

FIGURE 7.-Twelve-month running averages of critical frequencies plotted against 12-month running average of sunspot numbers. 
for the ionization. It should be noted that the noon $f_{E}$, the diurnal minimum $f^{x}$, and the noon $f^{x}{ }_{22}$, did not increase as rapidly with sunspot numbers near the maximum.

There is a trace of a lag on both ends of the $f^{x}$ and $f^{x}{ }_{F_{2}}$ curves, but not on the $f_{E}$ curve. It remains to be seen whether this effect persists throughout the descending half of the cycle or throughout subsequent cycles. It is well known that terrestrial magnetic disturbances are more frequent and severe following a sunspot maximum than preceding or during it. Since the seat of magnetic storms is principally in the $F_{2}$ region ${ }^{9}$ this is some indication that such a lag may be reasonable, and that the types of solar radiation responsible for $F_{2}$-layer ionization and magnetic storms may be related and may themselves reach a maximum after the sunspot numbers have started to decrease.

\section{CONCLUSIONS}

In conclusion, there is a good general correspondence, and, in the case of the $E$-layer, correlation in considerable detail, between the averages of sunspot numbers and the critical frequencies (or ionization densities), of the various ionosphere layers. This means that the averages of sunspot numbers give a good criterion of the general level of solar activity, and a good index to the general amount of ionizing radiation emitted from the sun. Exact correlation is indeed not to be expected in detail, since sunspots and the ionizing radiation are two different manifestations of solar activity, which follow the same trend in general but not in detail.

The existence of these relations, together with the vast amount of data available on sunspot activity in the past, suggests the possibility of forecasting the average condition of the ionosphere on the descending half of this cycle, and perhaps even in future cycles. Accordingly average radio-transmission conditions and average optimum frequencies for radio communication over different paths can be estimated, months and even years in advance.

Washington, August 30, 1938.

S. S. Kirby, T. R. Gilliland, E. B. Judson, N. Smith, The ionosphere, sunspots, and magnetic storms, Phys. Rev. 48,'849 (1935). 